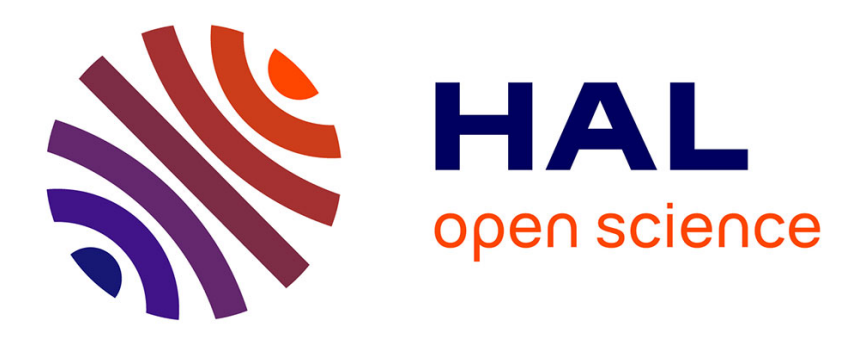

\title{
Cube polynomial of Fibonacci and Lucas cube
}

Sandi Klavzar, Michel Mollard

\section{- To cite this version:}

Sandi Klavzar, Michel Mollard. Cube polynomial of Fibonacci and Lucas cube. 2011. hal-00558273v2

\section{HAL Id: hal-00558273 \\ https://hal.science/hal-00558273v2}

Preprint submitted on 5 Oct 2011

HAL is a multi-disciplinary open access archive for the deposit and dissemination of scientific research documents, whether they are published or not. The documents may come from teaching and research institutions in France or abroad, or from public or private research centers.
L'archive ouverte pluridisciplinaire HAL, est destinée au dépôt et à la diffusion de documents scientifiques de niveau recherche, publiés ou non, émanant des établissements d'enseignement et de recherche français ou étrangers, des laboratoires publics ou privés. 


\title{
Cube polynomial of Fibonacci and Lucas cubes
}

\author{
Sandi Klavžar* \\ Faculty of Mathematics and Physics \\ University of Ljubljana, Slovenia \\ and \\ Faculty of Natural Sciences and Mathematics \\ University of Maribor, Slovenia \\ e-mail: sandi.klavzar@fmf.uni-lj.si \\ Michel Mollard \\ CNRS Université Joseph Fourier \\ Institut Fourier, BP 74 \\ 100 rue des Maths, 38402 St Martin d'Hères Cedex, France \\ e-mail: michel.mollard@ujf-grenoble.fr
}

\begin{abstract}
The cube polynomial of a graph is the counting polynomial for the number of induced $k$-dimensional hypercubes $(k \geq 0)$. We determine the cube polynomial of Fibonacci cubes and Lucas cubes, as well as the generating functions for the sequences of these cubes. Several explicit formulas for the coefficients of these polynomials are obtained, in particular they can be expressed with convolved Fibonacci numbers. Zeros of the studied cube polynomials are explicitly determined. Consequently, the coefficients sequences of cube polynomials of Fibonacci and Lucas cubes are unimodal.
\end{abstract}

Key words: hypercubes; cube polynomials; Fibonacci cubes; Lucas cubes; generating functions; zeros of polynomials, unimodal sequences

AMS subject classifications: 05C31, 05A15, 26C10

\section{Introduction}

The introduction of computers in the mid of the past century and the subsequent fascinating developments opened new horizons in the development of mathematics. In particular, numerous earlier sporadic fields of mathematics flourished and transformed themselves into mature fields. Let us just mention mathematical programming, theory of error-correcting codes, theory of computing, symbolic computation, and graph theory.

\footnotetext{
${ }^{*}$ Supported by the Research Grant P1-0297 of Ministry of Higher Education, Science and Technology Slovenia.
} 
One of the cornerstones of these developments is the binary number system. To build a related graph, it is utmost natural to let all binary strings of length $n, n \geq 1$, form the vertex set, and connect two vertices by an edge if they differ in precisely one coordinate (alias bit). The obtained graph is the $n$-dimensional hypercube or $n$-cube for short and denoted $Q_{n}$. The $n$-cubes are ubiquitous, say as the basic model for interconnection networks, a tool for numerous applications elsewhere, and a source of challenging problems is extremal combinatorics.

In this paper we are concerned with the enumeration of hypercubes in graphs, more precisely, in Fibonacci and Lucas cubes, two families of graphs of independent interest. So the primary task here is enumerative combinatorics, but a particular goal of this paper is to expose that for this task several areas of mathematics are useful: graph theory, number theory, complex analysis, and more.

For a graph $G$, let $c_{n}(G)(n \geq 0)$ be the number of induced subgraphs of $G$ isomorphic to $Q_{n}$. Note that in particular $c_{0}(G)=|V(G)|, c_{1}(G)=|E(G)|$, and $c_{2}(G)$ is the number of induced 4-cycles. The cube polynomial, $C(G, x)$, of $G$, is the corresponding counting polynomial, that is, the generating function

$$
C(G, x)=\sum_{n \geq 0} c_{n}(G) x^{n} .
$$

This polynomial was introduced in [4] where it was observed that it is multiplicative for the Cartesian multiplication of graphs: $C(G \square H, x)=C(G, x) C(H, x)$ holds for any graphs $G$ and $H$. Moreover, if $G$ is a median graph, then $C(G,-1)=1$ and $C^{\prime}(G,-1)$ is the smallest $n$ such that $G$ isometrically embeds into $Q_{n}$. The cube polynomial was generalized to a Hamming polynomial in two different ways in [3] and [7], respectively. For a survey on the cube polynomial, its extensions and related results see [16]. We add here that, interestingly, the sequence $\left\{c_{k}(G)\right\}_{k \geq 0}$ turned out to be important in human genetics, see [1] for its use in studies of the so-called phantom mutations.

Fibonacci cubes form appealing and applicable class of graphs introduced in the first place as a model for interconnection networks [10]. These graphs later found applications in mathematical chemistry $[15,28]$ and elsewhere. Fibonacci cubes were studied from algorithmic [9, 24] as well as from several other points of view, see [11] and references therein. Lucas cubes [18] form a class of graphs closely related to Fibonacci cubes, in a way they can be considered as a symmetrization of Fibonacci cubes.

There are (at least) three direct motivations for the present paper. In mathematical chemistry, the so-called resonance graphs play an important role because they reflect stability of molecules. It was proved in [15] that the resonance graphs of a special class of benzenoid graphs, fibonacenes, are precisely Fibonacci cubes. On the other hand, as proved in [21], the Clar number $C l(B)$ of a benzenoid system $B$ is equal to the number of subgraphs of the resonance graph of $B$ isomorphic to $Q_{C l(B)}$. Hence enumeration of hypercubes of all dimensions in Fibonacci cubes is essential there. Moreover, the same seems to be the case also with Lucas cubes because 
very recently it was proved in [29] that the resonance graphs of the so-called cyclic polyphenantrenes are precisely Lucas cube (and two additional isolated vertices).

Another motivation for us were several studied of enumerative properties of Fibonacci and Lucas cubes $[13,18,19]$. Considering the the cube polynomial of these cubes we in particular extend previous enumeration results. These results are presented in Sections 3-5.

Another motivation for our paper are the earlier investigations of zeros of cube polynomials of median graphs [5]. It was proved that $C(G, x)$ has no zeros in $[-1, \infty)$, that it always has a (real) zero in $[-2,-1)$, and that all rational zeros are of the form $-((t+1) / t)$ for some integer $t>0$. Moreover, if the cube polynomial $C(G, x)$ is of degree $p$, then $C(G, x)$ has a $p$-multiple zero if and only if $G$ is the Cartesian product of $p$ trees of the same order. Note that the smallest example for this theorem is provided with the fact that $C\left(Q_{p}, x\right)=(x+2)^{p}$. In the case of $Q_{3}$-free median graphs the cube polynomial is (of course) quadratic and it was proved [6] that its zeros are always real. In Section 6 we explicitly determine the zeros of the cube polynomial of the Fibonacci and Lucas cubes. It turns out that their zeros are real (and negative) which in turn implies that these polynomials are unimodal and log-concave.

\section{Preliminaries}

A Fibonacci string of length $n$ is a binary string $b_{1} b_{2} \ldots b_{n}$ with $b_{i} b_{i+1}=0$ for $1 \leq i<n$. The Fibonacci cube $\Gamma_{n}(n \geq 1)$ is the subgraph of $Q_{n}$ induced by the Fibonacci strings of length $n$. For convenience we also consider the empty string and set $\Gamma_{0}=K_{1}$. Call a Fibonacci string $b_{1} b_{2} \ldots b_{n}$ a Lucas string if $b_{1} b_{n}=0$. Then the Lucas cube $\Lambda_{n}(n \geq 1)$ is the subgraph of $Q_{n}$ induced by the Lucas strings of length $n$. We also set $\Lambda_{0}=K_{1}$. Note that every subcube of s Fibonacci cube or a Lucas cube is an induced subcube.

Let $\left\{F_{n}\right\}$ be the Fibonacci numbers: $F_{0}=0, F_{1}=1, F_{n}=F_{n-1}+F_{n-2}$ for $n \geq 2$. It is well known that the generating function of the sequence $\left\{F_{n}\right\}_{n=0}^{\infty}$ is

$$
\sum_{n \geq 0} F_{n} x^{n}=\frac{x}{1-x-x^{2}}
$$

Note that $\left|V\left(\Gamma_{n}\right)\right|=F_{n+2}$. We will denote by $\left\{\tilde{F}_{n}\right\}_{n=0}^{\infty}$ the sequence defined by $\tilde{F}_{n}=F_{n+1}$, hence

$$
\sum_{n \geq 0}\left(\tilde{F}_{n} x^{n}\right)=\frac{1}{1-x-x^{2}} .
$$

Let $\left\{L_{n}\right\}$ be the Lucas numbers: $L_{0}=2, L_{1}=1, L_{n}=L_{n-1}+L_{n-2}$ for $n \geq 2$. It is well known that the generating function of the sequence $\left\{L_{n}\right\}_{n=0}^{\infty}$ is

$$
\sum_{n \geq 0} L_{n} x^{n}=\frac{2-x}{1-x-x^{2}} .
$$


Note that $\left|V\left(\Lambda_{n}\right)\right|=L_{n}$ for $n \geq 1$.

Let $\left\{A_{n}\right\}_{n=0}^{\infty}$ and $\left\{B_{n}\right\}_{n=0}^{\infty}$ be two sequences of numbers, then the convolution of $A_{n}$ and $B_{n}$ is the sequence $\left\{(A * B)_{n}\right\}_{n=0}^{\infty}$ defined by $(A * B)_{n}=\sum_{i=0}^{n} A_{i} B_{n-i}$. ¿From the definition it is clear that the generating function of $\left\{(A * B)_{n}\right\}_{n=0}^{\infty}$ is the product of those of $\left\{A_{n}\right\}_{n=0}^{\infty}$ and $\left\{B_{n}\right\}_{n=0}^{\infty}$. We will denote by $A^{*}$ the sequence defined by $A^{*}=A$ and $A^{*}=A * A^{m}{ }^{*-1}(m \geq 2)$.

A classical question about a given sequence of numbers is whether it is unimodal, where a sequence $\left\{A_{n}\right\}_{n=0}^{m}$ of real numbers is unimodal if for some $0 \leq j \leq m$ we have $a_{0} \leq a_{1} \cdots \leq a_{j} \geq a_{j+1} \cdots \geq a_{m}$. A sequence $\left\{A_{n}\right\}_{n=0}^{m}$ of positive reals is said to be logarithmically concave (or log-concave for short) if $a_{i}^{2} \geq a_{i-1} a_{i+1}$ for all $1 \leq i \leq m$. It is easy to see that a positive log-concave sequence is unimodal, various additional methods are known for showing the unimodality or log-concavity of a sequence $[8,23,26]$. For our purposes it is important that by a result of Newton, if $\left\{A_{n}\right\}_{n=0}^{m}$ is a sequence of coefficients of a polynomial with negative real zeros, then $\left\{A_{n}\right\}_{n=0}^{m}$ is log-concave and therefore also unimodal, see [23, Theorem 2] (and the note after the theorem therein). A polynomial is log-concave (resp. unimodal) if the sequence of its coefficients is log-concave (resp. unimodal).

Finally, the Cartesian product $G \square H$ of graphs $G$ and $H$ has the vertex set $V(G) \times V(H)$, and $(g, h)$ is adjacent to $\left(g^{\prime}, h^{\prime}\right)$ if either $g=g^{\prime}$ and $h h^{\prime} \in E(H)$, or $g g^{\prime} \in E(G)$ and $h=h^{\prime}$.

\section{Cube polynomial of Fibonacci cubes}

In this section we determine the cube polynomial of Fibonacci cubes and read off the number of induced $Q_{k}$ in $\Gamma_{n}$. To get a feeling we list the first few of them:

$$
\begin{aligned}
& C\left(\Gamma_{0}, x\right)=1 \\
& C\left(\Gamma_{1}, x\right)=x+2 \\
& C\left(\Gamma_{2}, x\right)=2 x+3 \\
& C\left(\Gamma_{3}, x\right)=x^{2}+5 x+5 \\
& C\left(\Gamma_{4}, x\right)=3 x^{2}+10 x+8 \\
& C\left(\Gamma_{5}, x\right)=x^{3}+9 x^{2}+20 x+13 \\
& C\left(\Gamma_{6}, x\right)=4 x^{3}+22 x^{2}+38 x+21 \\
& C\left(\Gamma_{7}, x\right)=x^{4}+14 x^{3}+51 x^{2}+71 x+34 \\
& C\left(\Gamma_{8}, x\right)=5 x^{4}+40 x^{3}+111 x^{2}+130 x+55
\end{aligned}
$$

We next determine the generating function for the sequence of the corresponding cube polynomials:

Proposition 3.1 The generating function of the sequence $\left\{C\left(\Gamma_{n}, x\right)\right\}_{n=0}^{\infty}$ is

$$
\sum_{n \geq 0} C\left(\Gamma_{n}, x\right) y^{n}=\frac{1+y(1+x)}{1-y-y^{2}(1+x)} .
$$


Proof. Clearly, $C\left(\Gamma_{0}, x\right)=1$ and $C\left(\Gamma_{1}, x\right)=x+2$. Let $n \geq 2$ and let $X_{n}=\{x \in$ $\left.V\left(\Gamma_{n}\right) \mid x_{1}=0\right\}$ and $Y_{n}=\left\{x \in V\left(\Gamma_{n}\right) \mid x_{1}=1\right\}$. Then $X_{n}$ induces a subgraph of $\Gamma_{n}$ isomorphic to $\Gamma_{n-1}$. The first two coordinates of a vertex from $Y_{n}$ are 10, hence $Y_{n}$ induces a subgraph of $\Gamma_{n}$ isomorphic to $\Gamma_{n-2}$. Moreover, every vertex from $Y_{n}$ has exactly one neighbor in $X_{n}$ and these these edges form a matching. Hence for a subgraph $H$ of $\Gamma_{n}$ isomorphic to $Q_{k}$ we have exactly one of the following exclusive possibilities: (i) $H$ lies in the subgraph induced by $X_{n}$ or (ii) $H$ lies in the subgraph induced by $Y_{n}$ or (iii) $H=K \square K_{2}$, where $K$ is isomorphic to $Q_{k-1}$ and the edges of $K \square K_{2}$ corresponding to $K_{2}$ are edges between $X_{n}$ and $Y_{n}$. It follows that for $n \geq 2$,

$$
C\left(\Gamma_{n}, x\right)=C\left(\Gamma_{n-1}, x\right)+(1+x) C\left(\Gamma_{n-2}, x\right) \quad(n \geq 2) .
$$

Setting $f(x, y)=\sum_{n \geq 0} C\left(\Gamma_{n}, x\right) y^{n}$ we obtain $f(x, y)-1-y(x+2)=y(f(x, y)-$ $1)+y^{2}(1+x) f(x, y)$ and the result follows.

$Q_{n}$ can be represented as the Cartesian product of $n$ copies of $K_{2}$. Hence the property $C(G \square H, x)=C(G, x) C(H, x)$ immediately implies that for any $n \geq 0$,

$$
C\left(Q_{n}, x\right)=(2+x)^{n}=\sum_{a=0}^{n}\left(\begin{array}{l}
n \\
a
\end{array}\right)(1+x)^{a} .
$$

For Fibonacci cubes we have the following parallel result:

Theorem 3.2 For any $n \geq 0, C\left(\Gamma_{n}, x\right)$ is of degree $\left\lfloor\frac{n+1}{2}\right\rfloor$ and

$$
C\left(\Gamma_{n}, x\right)=\sum_{a=0}^{\left\lfloor\frac{n+1}{2}\right\rfloor}\left(\begin{array}{c}
n-a+1 \\
a
\end{array}\right)(1+x)^{a} .
$$


Proof. Using Proposition 3.1 we have:

$$
\begin{aligned}
\sum_{n \geq 0} C\left(\Gamma_{n}, x\right) y^{n} & =\frac{1+y(1+x)}{1-y-y^{2}(1+x)} \\
& =(1+y(1+x)) \sum_{a \geq 0}\left(y+y^{2}(1+x)\right)^{a} \\
& =\sum_{a \geq 0} y^{a}(1+y(1+x))^{a+1} \\
& =\sum_{a \geq 0} \sum_{b=0}^{a+1}\left(\begin{array}{c}
a+1 \\
b
\end{array}\right)(y(1+x))^{b} y^{a} \\
& =\sum_{a \geq 0} \sum_{b=0}^{a+1} \sum_{c=0}^{b}\left(\begin{array}{c}
a+1 \\
b
\end{array}\right)\left(\begin{array}{c}
b \\
c
\end{array}\right) x^{c} y^{a+b} \\
& =\sum_{a \geq 0} \sum_{n=a}^{2 a+1} \sum_{c=0}^{n-a}\left(\begin{array}{c}
a+1 \\
n-a
\end{array}\right)\left(\begin{array}{c}
n-a \\
c
\end{array}\right) x^{c} y^{n} \\
& =\sum_{n \geq 0}\left(\sum_{a \geq 0} \sum_{c=0}^{n-a}\left(\begin{array}{c}
a+1 \\
n-a
\end{array}\right)\left(\begin{array}{c}
n-a \\
c
\end{array}\right) x^{c}\right) y^{n} .
\end{aligned}
$$

¿From here we obtain

$$
\begin{aligned}
C\left(\Gamma_{n}, x\right) & =\sum_{a \geq 0} \sum_{c=0}^{n-a}\left(\begin{array}{c}
a+1 \\
n-a
\end{array}\right)\left(\begin{array}{c}
n-a \\
c
\end{array}\right) x^{c} \\
& =\sum_{a \geq 0}\left(\begin{array}{l}
a+1 \\
n-a
\end{array}\right) \sum_{c=0}^{n-a}\left(\begin{array}{c}
n-a \\
c
\end{array}\right) x^{c}=\sum_{a \geq 0}\left(\begin{array}{c}
a+1 \\
n-a
\end{array}\right)(1+x)^{n-a} \\
& =\sum_{a=0}^{n}\left(\begin{array}{c}
a+1 \\
n-a
\end{array}\right)(1+x)^{n-a}=\sum_{a=0}^{n}\left(\begin{array}{c}
n-a+1 \\
a
\end{array}\right)(1+x)^{a} \\
& =\sum_{a=0}^{\left\lfloor\frac{n+1}{2}\right\rfloor}\left(\begin{array}{c}
n-a+1 \\
a
\end{array}\right)(1+x)^{a},
\end{aligned}
$$

and we are done.

Corollary 3.3 For any $n \geq 0$, the number of induced $Q_{k}, k \geq 0$, in $\Gamma_{n}$ is

$$
c_{k}\left(\Gamma_{n}\right)=\sum_{i=k}^{\left\lfloor\frac{n+1}{2}\right\rfloor}\left(\begin{array}{c}
n-i+1 \\
i
\end{array}\right)\left(\begin{array}{l}
i \\
k
\end{array}\right) .
$$


Like the determination of $c_{k}\left(Q_{n}\right)$ in [14], Corollary 3.3 can also be deduced directly as follows. Note first that there are precisely $\left(\begin{array}{c}n-i+1 \\ i\end{array}\right)$ Fibonacci strings that contain $i$ 1's. (Indeed, writing down $n-i$ zeros, we need to select among $n-i+1$ positions where to insert ones.) For a given subcube $Q_{k}$ of $\Gamma_{n}$, let $u$ be the vertex of the $Q_{k}$ that contains the largest number of 1 's, say $i$, among the vertices of the $Q_{k}$. Then there is set of $k$ positions such that the $Q_{k}$ is induced by the $2^{k}$ vertices obtained by varying these $k$ bits. Now, each such vertex $u$ give rise to $\left(\begin{array}{l}i \\ k\end{array}\right)$ different induced $k$-cubes and the result follows.

\section{Fibonacci cubes and convolved Fibonacci numbers}

We next determine, for a fixed $k$, the generating function of the sequence $\left\{c_{k}\left(\Gamma_{n}\right)\right\}_{n=0}^{\infty}$ :

Theorem 4.1 Let $k \geq 1$ be a fixed integer. Then

$$
\sum_{n \geq 0} c_{k}\left(\Gamma_{n}\right) y^{n}=\frac{y^{2 k-1}}{\left(1-y-y^{2}\right)^{k+1}} .
$$

Proof. Since $c_{0}\left(\Gamma_{n}\right)=\left|V\left(\Gamma_{n}\right)\right|=F_{n+2}$, we have

$$
\sum_{n \geq 0} c_{0}\left(\Gamma_{n}\right) y^{n}=\frac{1+y}{1-y-y^{2}} .
$$

As in the proof of Proposition 3.1, let $n \geq 2$ and consider the partition of $V\left(\Gamma_{n}\right)$ into the sets $X_{n}=\left\{x \in V\left(\Gamma_{n}\right) \mid x_{1}=0\right\}$ and $Y_{n}=\left\{x \in V\left(\Gamma_{n}\right) \mid x_{1}=1\right\}$ to infer that a subgraph $H$ of $\Gamma_{n}$ isomorphic to $Q_{k}$ either lies in the subgraph induced by $X_{n}$, or it lies in the subgraph induced by $Y_{n}$, or it is of the form $K \square K_{2}$ with $K=Q_{k-1}$ and the edges of $K \square K_{2}$ corresponding to $K_{2}$ are edges between $X_{n}$ and $Y_{n}$. Therefore,

$$
c_{k}\left(\Gamma_{n}\right)=c_{k}\left(\Gamma_{n-1}\right)+c_{k}\left(\Gamma_{n-2}\right)+c_{k-1}\left(\Gamma_{n-2}\right) .
$$

Since $c_{1}\left(\Gamma_{0}\right)=0$ and $c_{1}\left(\Gamma_{1}\right)=1$, a routine computation, using (3) and (2), yields

$$
\sum_{n \geq 0} c_{1}\left(\Gamma_{n}\right) y^{n}=\frac{y}{\left(1-y-y^{2}\right)^{2}} .
$$

To rich the conclusion for $k \geq 2$, apply induction, the fact that $c_{k}\left(\Gamma_{0}\right)=c_{k}\left(\Gamma_{1}\right)=0$, and (3).

Corollary 4.2 Let $k \geq 0$. Then the number of induced $Q_{k}$ in $\Gamma_{n}$ is

$$
c_{k}\left(\Gamma_{n}\right)=\tilde{F}_{n-2 k+1}^{k+1}
$$


Proof. Since $\sum_{n \geq 0}\left(\tilde{F}_{n} y^{n}\right)=\frac{1}{1-y-y^{2}}$, we have $\sum_{n \geq 0}\left(\tilde{F}_{n}{ }^{k+1} y^{n}\right)=\frac{1}{\left(1-y-y^{2}\right)^{k+1}}$. So the coefficient at $y^{n}$ in the expansion of $\frac{1}{\left(1-y-y^{2}\right)^{k+1}}$ is $\tilde{F}_{n}^{k+1}$ and hence the coefficient at $y^{n}$ in the expansion of $\frac{y^{2 k-1}}{\left(1-y-y^{2}\right)^{k+1}}$ is $\tilde{F}_{n-2 k+1}^{k+1}$. The assertion follows by Theorem 4.1.

Note that Corollary 4.2 for $k=0$ reads as $\left|V\left(\Gamma_{n}\right)\right|=c_{0}\left(\Gamma_{n}\right)=\tilde{F}_{n+1}^{1}=\tilde{F}_{n+1}=$ $F_{n+2}$.

The numbers $\tilde{F}_{n}^{*}$ are known as convolved Fibonacci numbers and have been studied earlier, for the first time probably in [20]. Bergum and Hoggatt [2] considered the array $R_{n, m}$ for $m \geq 1, n \geq 1$ whose $m^{\text {th }}$ column is the sequence $\left\{\tilde{F}_{n-1}^{*}\right\}_{n=1}^{\infty}$. Thus $c_{k}\left(\Gamma_{n}\right)=R_{n-2 k+2, k+1}$ and the coefficients of $C\left(\Gamma_{n}, x\right)$ appear as rising diagonal of this array:

$$
C\left(\Gamma_{n}, x\right)=\sum_{k=0}^{\left\lfloor\frac{n+1}{2}\right\rfloor} R_{n-2 k+2, k+1} x^{k} .
$$

They also proved that for any $n \geq 1, \sum_{m \geq 1} R_{n, m} x^{m-1}=\frac{N_{n}(x)}{(1-x)^{n}}$ where

$$
N_{n}(x)=\sum_{k=0}^{\left\lfloor\frac{n-1}{2}\right\rfloor} R_{n-2 k, k+1}(-1)^{k} x^{k}
$$

Furthermore, they studied the properties of the polynomial $N_{n}(x)$ that differs from $C\left(\Gamma_{n-2}, x\right)$ by the alternate sign of coefficients. The same array appears also in $[17$, 12] with the notation $F_{n+1}^{(r)}=\tilde{F}_{n}^{*}$; see [22] for additional references. (Note that we selected the notation with reference to convolution because it can be also used for Lucas cubes; see below.)

Using the properties of convolved Fibonacci numbers, we can state further results on the number of cubes in Fibonacci cubes. For instance, since the convolved Fibonacci number can be written as sums of products of Fibonacci numbers, see [17, page 1] (this fact can also be deduced directly from the definition of convolution):

Corollary 4.3 For any $n \geq 1$,

$$
c_{k}\left(\Gamma_{n}\right)=\sum_{\substack{i_{1}, i_{2}, \ldots, i_{k+1} \\ i_{1}+i_{2}+\cdots+i_{k+1}=n-2 k+1}} F_{i_{1}+1} F_{i_{2}+1} \cdots F_{i_{k+1}+1} .
$$

Corollary 4.3 for $k=1$ reduces to

$$
\left|E\left(\Gamma_{n}\right)\right|=c_{1}\left(\Gamma_{n}\right)=\sum_{\substack{i, j \\ i+j=n-1}} F_{i+1} F_{j+1}=\sum_{i=0}^{n-1} F_{i+1} F_{n-i}=\sum_{i=1}^{n} F_{i} F_{n-i+1},
$$


a result obtained in [13, Proposition 3], while for the squares $S\left(\Gamma_{n}\right)$ of $\Gamma_{n}$ it gives:

$$
\left|S\left(\Gamma_{n}\right)\right|=c_{2}\left(\Gamma_{n}\right)=\sum_{\substack{i, j, k \\ i+j+k=n-3}} F_{i+1} F_{j+1} F_{k+1} .
$$

Another way of looking at Corollary 4.3 is:

Corollary 4.4 For any $n \geq 1$,

$$
c_{k}\left(\Gamma_{n}\right)=\sum_{i=1}^{n-2 k+2} F_{i} c_{k-1}\left(\Gamma_{n-i-1}\right) .
$$

The case $k=2$ of Corollary 4.4 reduces to [13, Proposition 6].

\section{$5 \quad$ Lucas cubes}

In this section we consider Lucas cubes and present results parallel to the results for Fibonacci cubes from Sections 3 and 4. Proofs are similar as in the previous sections hence we omit most of the details but point out the differences. The first polynomials are:

$$
\begin{aligned}
& C\left(\Lambda_{0}, x\right)=1 \\
& C\left(\Lambda_{1}, x\right)=1 \\
& C\left(\Lambda_{2}, x\right)=2 x+3 \\
& C\left(\Lambda_{3}, x\right)=3 x+4 \\
& C\left(\Lambda_{4}, x\right)=2 x^{2}+8 x+7 \\
& C\left(\Lambda_{5}, x\right)=5 x^{2}+15 x+11 \\
& C\left(\Lambda_{6}, x\right)=2 x^{3}+15 x^{2}+30 x+18 \\
& C\left(\Lambda_{7}, x\right)=7 x^{3}+35 x^{2}+56 x+29 \\
& C\left(\Lambda_{8}, x\right)=2 x^{4}+24 x^{3}+80 x^{2}+104 x+47
\end{aligned}
$$

We begin with the generating function for the sequence of the cube polynomials of Lucas cubes:

Proposition 5.1 The generating function of the sequence $\left\{C\left(\Lambda_{n}, x\right)\right\}_{n=0}^{\infty}$ is

$$
\sum_{n \geq 0} C\left(\Lambda_{n}, x\right) y^{n}=\frac{1+y^{2}(1+x)}{1-y-y^{2}(1+x)} .
$$

Proof. Let $n \geq 3$ and partition the vertex set of $\Lambda_{n}$ into vertices that start with 0 and those that start with 1 . The latter vertices are then of the form $10 \ldots 0$. Similarly as in the proof of Proposition 3.1 we now get that for $n \geq 3$,

$$
C\left(\Lambda_{n}, x\right)=C\left(\Gamma_{n-1}, x\right)+(1+x) C\left(\Gamma_{n-3}, x\right) .
$$


To complete the proof combine Proposition 3.1 with (4) and the initial conditions $C\left(\Lambda_{0}, x\right)=C\left(\Lambda_{1}, x\right)=1, C\left(\Lambda_{2}, x\right)=2 x+3$.

We note in passing that considering degree zero coefficients in (4) yields the well known relation $L_{n}=F_{n+1}+F_{n-1}$.

Theorem 5.2 For any $n \geq 3, C\left(\Lambda_{n}, x\right)$ is of degree $\left\lfloor\frac{n}{2}\right\rfloor$ and

$$
C\left(\Lambda_{n}, x\right)=\sum_{a=0}^{\left\lfloor\frac{n}{2}\right\rfloor}\left[2\left(\begin{array}{c}
n-a \\
a
\end{array}\right)-\left(\begin{array}{c}
n-a+1 \\
a
\end{array}\right)\right](1+x)^{a} .
$$

Proof. Rewrite (1) as $C\left(\Gamma_{n-1}, x\right)=C\left(\Gamma_{n-2}, x\right)+(1+x) C\left(\Gamma_{n-3}, x\right)$ and subtract this equality from (4) to obtain

$$
C\left(\Lambda_{n}, x\right)=2 C\left(\Gamma_{n-1}, x\right)-C\left(\Gamma_{n-2}, x\right) \quad(n \geq 3) .
$$

The result now follows from Theorem 3.2.

Corollary 5.3 Let $n \geq 1$. Then the number of induced $Q_{k}, k \geq 1$, in $\Lambda_{n}$ is

$$
c_{k}\left(\Lambda_{n}\right)=\sum_{i=k}^{\left\lfloor\frac{n}{2}\right\rfloor}\left[2\left(\begin{array}{c}
n-i \\
i
\end{array}\right)-\left(\begin{array}{c}
n-i+1 \\
i
\end{array}\right)\right]\left(\begin{array}{l}
i \\
k
\end{array}\right) .
$$

We continue with the generating function of the sequence $\left\{c_{k}\left(\Lambda_{n}\right)\right\}_{n=0}^{\infty}$, where $k$ is a fixed integer.

Theorem 5.4 Let $k \geq 1$ be a fixed integer. Then

$$
\sum_{n \geq 0} c_{k}\left(\Lambda_{n}\right) y^{n}=\frac{y^{2 k}(2-y)}{\left(1-y-y^{2}\right)^{k+1}} .
$$

Proof. From (4) we deduce that for $n \geq 3$,

$$
c_{k}\left(\Lambda_{n}\right)=c_{k}\left(\Gamma_{n-1}\right)+c_{k}\left(\Gamma_{n-3}\right)+c_{k-1}\left(\Gamma_{n-3}\right) .
$$

Assume first $k \geq 2$. Then $c_{k}\left(\Lambda_{2}\right)=c_{k}\left(\Lambda_{1}\right)=c_{k}\left(\Lambda_{0}\right)=c_{k}\left(\Gamma_{1}\right)=c_{k}\left(\Gamma_{0}\right)=0$. Then, using Theorem 4.1, we can compute as follows:

$$
\begin{aligned}
\sum_{n \geq 0} c_{k}\left(\Lambda_{n}\right) y^{n} & =\sum_{n \geq 3} c_{k}\left(\Lambda_{n}\right) y^{n} \\
& =y \sum_{n \geq 3} c_{k}\left(\Gamma_{n-1}\right) y^{n-1}+y^{3} \sum_{n \geq 3} c_{k}\left(\Gamma_{n-3}\right) y^{n-3}+y^{3} \sum_{n \geq 3} c_{k-1}\left(\Gamma_{n-3}\right) y^{n-3} \\
& =y \sum_{n \geq 0} c_{k}\left(\Gamma_{n}\right) y^{n}+y^{3} \sum_{n \geq 0} c_{k}\left(\Gamma_{n}\right) y^{n}+y^{3} \sum_{n \geq 0} c_{k-1}\left(\Gamma_{n}\right) y^{n} \\
& =y \frac{y^{2 k-1}}{\left(1-y-y^{2}\right)^{k+1}}+y^{3} \frac{y^{2 k-1}}{\left(1-y-y^{2}\right)^{k+1}}+y^{3} \frac{y^{2 k-3}}{\left(1-y-y^{2}\right)^{k}} \\
& =\frac{y^{2 k}(2-y)}{\left(1-y-y^{2}\right)^{k+1}} .
\end{aligned}
$$


Consider now the case $k=1$. Because $c_{1}\left(\Lambda_{1}\right)=c_{1}\left(\Lambda_{0}\right)=0$ we have

$$
\sum_{n \geq 0} c_{1}\left(\Lambda_{n}\right) y^{n}=\sum_{n \geq 3} c_{1}\left(\Lambda_{n}\right) y^{n}+2 y^{2}
$$

and

$$
\sum_{n \geq 3} c_{1}\left(\Lambda_{n}\right) y^{n}=y \sum_{n \geq 3} c_{1}\left(\Gamma_{n-1}\right) y^{n-1}+y^{3} \sum_{n \geq 3} c_{1}\left(\Gamma_{n-3}\right) y^{n-3}+y^{3} \sum_{n \geq 3} c_{0}\left(\Gamma_{n-3}\right) y^{n-3} .
$$

We now determine the latter terms using Theorem 4.1 and (2) as follows:

$$
\begin{aligned}
\sum_{n \geq 3} c_{1}\left(\Gamma_{n-1}\right) y^{n-1} & =\sum_{n \geq 0} c_{1}\left(\Gamma_{n}\right) y^{n}-c_{1}\left(\Gamma_{1}\right) y-c_{1}\left(\Gamma_{0}\right)=\frac{y}{\left(1-y-y^{2}\right)^{2}}-y \\
\sum_{n \geq 3} c_{1}\left(\Gamma_{n-3}\right) y^{n-3} & =\sum_{n \geq 0} c_{1}\left(\Gamma_{n}\right) y^{n}=\frac{y}{\left(1-y-y^{2}\right)^{2}}, \\
\sum_{n \geq 3} c_{0}\left(\Gamma_{n-3}\right) y^{n-3} & =\frac{1+y}{1-y-y^{2}}=\frac{y^{-1}}{1-y-y^{2}}-y^{-1} .
\end{aligned}
$$

Thus

$$
\sum_{n \geq 0} c_{1}\left(\Lambda_{n}\right) y^{n}=\frac{y^{2}(2-y)}{\left(1-y-y^{2}\right)^{2}}
$$

Corollary 5.5 Let $k \geq 1$. Then the number of induced $Q_{k}$ in $\Lambda_{n}$ is

$$
c_{k}\left(\Lambda_{n}\right)=\left(L * \tilde{F}^{k}\right)_{n-2 k} .
$$

Proof. The generating function of $L * \tilde{F}^{k}$ is $\frac{2-y}{1-y-y^{2}} \cdot \frac{1}{\left(1-y-y^{2}\right)^{k}}$. Then arguing similarly as in the proof of Corollary 4.2 the result follows.

Proceeding along the same lines as at the end of Section 4 we have:

Corollary 5.6 For any $k \geq 1$,

$$
c_{k}\left(\Lambda_{n}\right)=\sum_{\substack{i_{1}, i_{2}, \ldots, i_{k+1} \\ i_{1}+i_{2}+\cdots+i_{k+1}=n-2 k}} L_{i_{1}} F_{i_{2}+1} \ldots F_{i_{k+1}+1}=\sum_{i=0}^{n-2 k} L_{i} c_{k-1}\left(\Gamma_{n-i-3}\right) .
$$

The first equality of Corollary 5.6 reduces for $k=1$ to [13, Proposition 7], while the second equality for $k=2$ was obtained in [13, Proposition 8]. 


\section{Roots and unimodality of $C\left(\Gamma_{n}, x\right)$ and $C\left(\Lambda_{n}, x\right)$}

To present Binet-like formulas for $C\left(\Gamma_{n}, x\right)$ and $C\left(\Lambda_{n}, x\right)$, the following recursion is useful:

Lemma 6.1 For any $n \geq 3, C\left(\Lambda_{n}, x\right)=C\left(\Lambda_{n-1}, x\right)+(1+x) C\left(\Lambda_{n-2}, x\right)$.

Proof. Follows from relations (1) and (4) and considering small values of $n$ separately.

Theorem 6.2 Let $x \neq-5 / 4$. Then

$$
\begin{aligned}
& \text { (i) } C\left(\Gamma_{n}, x\right)=\frac{1}{\sqrt{5+4 x}}\left[\left(\frac{1+\sqrt{5+4 x}}{2}\right)^{n+2}-\left(\frac{1-\sqrt{5+4 x}}{2}\right)^{n+2}\right] \quad(n \geq 0) \text {, } \\
& \text { (ii) } C\left(\Lambda_{n}, x\right)=\left(\frac{1+\sqrt{5+4 x}}{2}\right)^{n}+\left(\frac{1-\sqrt{5+4 x}}{2}\right)^{n} \quad(n \geq 1) .
\end{aligned}
$$

Proof. Theorem is proved by standard methods for second order linear recursion, or by induction: for (i) use (1) together with initial conditions and for (ii) apply Lemma 6.1 together with initial conditions.

When $x=-5 / 4$ we obtain by continuity (or with the standard method when the roots of the characteristic polynomial are equals) that

$$
C\left(\Gamma_{n},-5 / 4\right)=\frac{n+2}{2^{n+1}}
$$

and

$$
C\left(\Lambda_{n},-5 / 4\right)=\frac{1}{2^{n-1}}(n \geq 1) .
$$

Theorem 6.2 leads to numerous divisibility consequences, some are collected in the next statement.

Corollary 6.3 (i) For any $p \geq 1, C\left(\Gamma_{2 p}, x\right)=C\left(\Gamma_{p-1}, x\right) C\left(\Lambda_{p+1}, x\right)$.

(ii) For any $n, k \geq 0, C\left(\Gamma_{n}, x\right)$ divides $C\left(\Gamma_{k(n+2)+n}, x\right)$.

(iii) For any $n, k \geq 1, C\left(\Lambda_{n}, x\right)$ divides $C\left(\Lambda_{k n}, x\right)$.

Proof. Set $f(x)=\frac{1+\sqrt{5+4 x}}{2}$ and $g(x)=\frac{1-\sqrt{5+4 x}}{2}$.

(i) By Theorem 6.2,

$$
\begin{aligned}
C\left(\Gamma_{2 p}, x\right) & =\frac{1}{\sqrt{5+4 x}}\left[f^{2 p+2}(x)-g^{2 p+2}(x)\right] \\
& =\frac{1}{\sqrt{5+4 x}}\left[f^{p+1}(x)-g^{p+1}(x)\right]\left[f^{p+1}(x)+g^{p+1}(x)\right] \\
& =C\left(\Gamma_{p-1}, x\right) C\left(\Lambda_{p+1}, x\right) .
\end{aligned}
$$


(ii) Suppose $C\left(\Gamma_{n}, x_{0}\right)=0$. Then by Theorem 6.2, $f^{n+2}\left(x_{0}\right)=g^{n+2}\left(x_{0}\right)$. It follows that $f^{(k+1)(n+2)}\left(x_{0}\right)=g^{(k+1)(n+2)}\left(x_{0}\right)$ which in turn implies that $C\left(\Gamma_{k(n+2)+2}, x_{0}\right)=0$.

(iii) $C\left(\Lambda_{n}, x_{0}\right)=0$ means that $f^{n}\left(x_{0}\right)=-g^{n}\left(x_{0}\right)$. Therefore $f^{k n}\left(x_{0}\right)=-g^{k n}\left(x_{0}\right)$ which in turn implies $C\left(\Lambda_{k n}, x_{0}\right)=0$.

In order to establish unimodality of coefficients of Fibonacci and Lucas polynomials, we next explicitly determine its zeros.

Proposition 6.4 Let $n \geq 1$. Then the roots of $C\left(\Gamma_{n}, x\right)$ and $C\left(\Lambda_{n, x}\right)$ are

$$
-\frac{\tan ^{2}\left(\frac{r \pi}{n+2}\right)+5}{4}, \quad r=1,2, \ldots,\left\lfloor\frac{n+1}{2}\right\rfloor,
$$

and

$$
-\frac{\tan ^{2}\left(\frac{(2 r+1) \pi}{2 n}\right)+5}{4}, \quad r=0,1, \ldots,\left\lfloor\frac{n}{2}\right\rfloor-1,
$$

respectively. In particular, all the roots are negative reals.

Proof. By Theorem 6.2, roots of $C\left(\Gamma_{n}, x\right)$ are determined with

$$
(1+\sqrt{5+4 x})^{n+2}=(1-\sqrt{5+4 x})^{n+2} .
$$

Suppose $x \in \mathbb{R}$. Then for a root, $5 x+4<0$ must hold. Writing $\sqrt{5+4 x}=i y$ we need to solve $(1+i y)^{n+2}=(1-i y)^{n+2}$. Setting $z=1+i y=|z|(\cos \phi+i \sin \phi)$ this reduces to $\sin (n+2) \phi=0$ so that $y=\tan \phi=\tan \left(\frac{k \pi}{n+2}\right)$. Hence the zeros. Note that the above zeros are all different because $\tan ^{2}(x)$ is strictly increasing on $[0, \pi / 2)$.

The degree of $C\left(\Gamma_{n}, x\right)$ is $\left\lfloor\frac{n+1}{2}\right\rfloor$ thus we conclude that all zeros are negative reals.

Similar computation gives the zeros of $C\left(\Lambda_{n}, x\right)$.

The sequence of coefficients of a polynomial whose zeros are all negative reals is $\log$-concave and a positive log-concave sequence is unimodal $[8,23,26]$. Hence:

Corollary 6.5 For any $n \geq 0$, the sequences of coefficients of $C\left(\Gamma_{n}, x\right)$ and $C\left(\Lambda_{n}, x\right)$ are log-concave and unimodal.

\section{Concluding remark}

For $n \geq i \geq 0$, the $i$ th extended Fibonacci cube of order $n, \Gamma_{n}^{i}$, was introduced in [27] as follows. Let $B_{i}$ be the set of all binary strings of length $i$. Then the vertex set $V_{n}^{i}$ of $\Gamma_{n}^{i}$ is defined recursively by $V_{n+2}^{i}=0 V_{n+1}^{i} \cup 10 V_{n}^{i}$, with initial conditions $V_{i}^{i}=B_{i}, V_{i+1}^{i}=B_{i+1}$. Note that then $\Gamma_{i}^{i}=Q_{i}$ and $\Gamma_{i+1}^{i}=Q_{i+1}$. Observe also that $\Gamma_{n}^{0}=\Gamma_{n}$. It was proved in [25] (see also [13]), that $\Gamma_{n}^{i}$ is isomorphic to $\Gamma_{n-i} \square Q_{i}$. Hence understanding the cube polynomial of Fibonacci cubes and how it behaves under the Cartesian multiplication, one can obtain all the corresponding results for the extended Fibonacci cubes as well. 


\section{References}

[1] H.-J. Bandelt, L. Quintana-Murci, A. Salas, and V. Macaulay. The fingerprint of phantom mutations in mitochondrial DNA data. Amer. J. Human Gen., 71:1150-1160, 2002.

[2] G. E. Bergum and V. E. Hoggatt, Jr. Numerator polynomial coefficient array for the convolved Fibonacci sequence. Fibonacci Quart, 14(1):43-48, 1976.

[3] B. Brešar, P. Dorbec, S. Klavžar, and M. Mollard. Hamming polynomials and their partial derivatives. European J. Combin., 28(4):1156-1162, 2007.

[4] B. Brešar, S. Klavžar, and R. Škrekovski. The cube polynomial and its derivatives: the case of median graphs. Electron. J. Combin., 10:Research Paper 3, 11 pp. (electronic), 2003.

[5] B. Brešar, S. Klavžar, and R. Škrekovski. Roots of cube polynomials of median graphs. J. Graph Theory, 52(1):37-50, 2006.

[6] B. Brešar, S. Klavžar, and R. Škrekovski. On cube-free median graphs. Discrete Math., 307(3-5):345-351, 2007.

[7] B. Brešar and A. Tepeh Horvat. Cage-amalgamation graphs, a common generalization of chordal and median graphs. European J. Combin., 30(5):1071-1081, 2009.

[8] L. Comtet. Advanced Combinatorics. D. Reidel Publishing Co., Dordrecht, enlarged edition, 1974.

[9] P. Gregor. Recursive fault-tolerance of Fibonacci cube in hypercubes. Discrete Math., 306(13):1327-1341, 2006.

[10] W.-J. Hsu. Fibonacci cubes - a new interconnection technology. IEEE Trans. Parallel Distrib. Syst., 4(1):3-12, 1993.

[11] A. Ilić, S. Klavžar, and Y. Rho. Generalized Fibonacci cubes. To appear in Discrete Math., 2011.

[12] M. Janjić. Number of compositions and convolved Fibonacci numbers. arXiv:1003.0981, 2010.

[13] S. Klavžar. On median nature and enumerative properties of Fibonacci-like cubes. Discrete Math., 299(1-3):145-153, 2005.

[14] S. Klavžar. Counting hypercubes in hypercubes. Discrete Math., 306(22):29642967, 2006.

[15] S. Klavžar and P. Žigert. Fibonacci cubes are the resonance graphs of Fibonaccenes. Fibonacci Quart., 43(3):269-276, 2005. 
[16] M. Kovše. Complexity of phylogenetic networks: counting cubes in median graphs and related problems. In Analysis of Complex Networks: From Biology to Linguistics, pages 323-350. WILEY-VCH, Weinheim, 2009.

[17] P. Moree. Convoluted convolved Fibonacci numbers. J. Integer Seq., 7(2):Article 04.2.2, 14 pp. (electronic), 2004.

[18] E. Munarini, C. Perelli Cippo, and N. Zagaglia Salvi. On the Lucas cubes. Fibonacci Quart., 39(1):12-21, 2001.

[19] E. Munarini and N. Salvi Zagaglia. Structural and enumerative properties of the Fibonacci cubes. Discrete Math., 255(1-3):317-324, 2002.

[20] J. Riordan. Combinatorial Identities. John Wiley \& Sons Inc., New York, 1968.

[21] K. Salem, S. Klavžar, A. Vesel, and P. Žigert. The Clar formulas of a benzenoid system and the resonance graph. Discrete Appl. Math., 157(11):2565-2569, 2009.

[22] N. J. A. Sloane. The On-Line Encyclopedia of Integer Sequences. Published electronically at http://oeis.org, 2011.

[23] R. P. Stanley. Log-concave and unimodal sequences in algebra, combinatorics, and geometry. In Graph Theory and its Applications: East and West (Jinan, 1986), volume 576 of Ann. New York Acad. Sci., pages 500-535. New York Acad. Sci., New York, 1989.

[24] A. Taranenko and A. Vesel. Fast recognition of Fibonacci cubes. Algorithmica, 49(2):81-93, 2007.

[25] C. Whitehead and N. Zagaglia Salvi. Observability of the extended Fibonacci cubes. Discrete Math., 266(1-3):431-440, 2003. The 18th British Combinatorial Conference (Brighton, 2001).

[26] H. S. Wilf. generatingfunctionology. A K Peters Ltd., Wellesley, MA, third edition, 2006.

[27] J. Wu. Extended Fibonacci cubes. IEEE Trans. Parallel Distr. Systems, 8:3-9, 1997.

[28] H. Zhang, L. Ou, and H. Yao. Fibonacci-like cubes as Z-transformation graphs. Discrete Math., 309(6):1284-1293, 2009.

[29] P. Žigert and M. Berlič. Lucas cubes and resonance graphs of cyclic polyphenantrenes. Manuscript, 2011. 